\title{
IRREDUNDANT SETS IN BOOLEAN ALGEBRAS
}

\author{
STEVO TODORCEVIC
}

\begin{abstract}
It is shown that every uncountable Boolean algebra $A$ contains an uncountable subset $I$ such that no $a$ of $I$ is in the subalgebra generated by $I \backslash\{a\}$ using an additional axiom of set theory. It is also shown that a use of some such axiom is necessary.
\end{abstract}

A subset $I$ of a Boolean algebra $A$ is irredundant if no proper subset of $I$ generates the same subalgebra as $I$, or equivalently, if no $a$ of $I$ is in the subalgebra generated by $I \backslash\{a\}$. Typical irredundant subsets of $A$ are chains of $A$, i.e., subsets which are totally ordered by the inclusion. Other types of irredundant subsets of $A$ include infinite subsets of $A$ of pairwise disjoint elements or infinite subsets $I$ of $A$ which are ideal-independent, i.e., no element of $I$ can be covered by finitely many others. Note that these two types of irredundant sets are similar in nature in the sense that an ideal-independent set reduces to a disjoint family in some quotient of $A$. It is natural to expect that large Boolean algebras (BA's) have large irredundant sets and, in particular, that uncountable BA's have uncountable irredundant sets (a question that has been explicitly asked in [2, Q21]). One may go further and ask whether every uncountable Boolean algebra has either an uncountable ideal independent subset or an uncountable chain or, more reasonably, an uncountable chain in one of its quotients (see [2, ff4, p. 240] where the stronger form of the question has been explicitly asked). In this form the problem reduces to a well-known problem about the structure of perfectly normal compacta which has many ramifications (see [3]). The algebraic approach to the problem gives more information about its nature and various connections while its dual form gives us more hints about methods which might be relevant for its solution. It should be pointed out, however, that if we ask for an uncountable irredundant set the problem has no reasonable dual form and is, in some sense, purely algebraic. The purpose of this note is to present an axiomatic analysis of the problem using the language of the Proper Forcing Axiom. We shall assume the reader is familiar with Chapter 8 of [9] which discusses a method of building proper partial orderings that will be used here. Our principal result has the following form.

Theorem 1 (PFA). Every uncountable Boolean algebra has an uncountable irredundant subset.

Received by the editors June 11, 1990 and, in revised form, November 24, 1990. 1980 Mathematics Subject Classification (1985 Revision). Primary 03E50, 06 E05. Research supported in part by NSF Grant DMS-8505550. 
The proof will be given in a sequence of claims and lemmas. Let $A$ be a fixed uncountable BA and let $X$ be the dual space of $A$. A subset $I$ of $A$ is left-separated if there is a well-ordering < of $I$ such that no $a$ in $I$ can be covered by finitely many elements of $I$ which are $<a$. The full strength of the following lemma will not be needed for the proof of Theorem 1 and it is given here only because it gives a good introduction into more complex arguments that will be used later.

Lemma 1 (PFA). Suppose I is an uncountable left-separated subset of $A$. Then there is an uncountable set $J$ of finite unions of elements of $I$ that is irredundant in $A$.

Proof. We may assume that $I,<$ has order type $\omega_{1}$. For $a$ in $I$ fix $x_{a}$ in $X$ such that $x_{a} \in a$ but $x_{a} \notin b$ for all $b<a$ in $I$. Define now $[I]^{2}=K_{0} \cup K_{1}$ by letting $\{a, b\}_{<}$in $K_{0}$ if $x_{a}$ is not an element of $b$. By the partition property $(P)$ of $[9, \S 9]$ there is either an uncountable 0 -homogeneous subset of $I$ (which will clearly be an ideal-independent set), or else uncountable $H \subseteq I$ and disjoint uncountable $\mathscr{F} \subseteq[I]^{<\omega}$ such that for all $a$ in $H$ and $F$ in $\mathscr{F}$ above $a$ there is $b$ in $F$ such that $x_{a} \in b$. If the second alternative occurs, for $F$ in $\mathscr{F}$ let $b_{F}$ be the union of the elements of $F$. Then $J=\left\{b_{F}: F \in \mathscr{F}\right\}$ is an uncountable irredundant subset of $A$ assuming (which we may) that elements of $\mathscr{F}$ are <-increasing in order and that for every $F<G$ in $\mathscr{F}$ there is an $a$ in $H$ such that $F<a<G$. This completes the proof.

The consequence of Lemma 1 that will be used in the rest of the proof of Theorem 1 is that we may assume that every ideal of our algebra $A$ is countably generated. Another observation we shall need frequently is that if $I \subset A$ is right-separated (i.e., no $a$ in $I$ can be covered by finitely many elements of $I$ above $a$ ) then we can find $J \subseteq I$ of the same size as $I$ such that $J$ is irredundant in $A$. Hence we may assume that our algebra $A$ contains no uncountable left- nor right-separated subset. We can also assume $A$ has size $\aleph_{1}$ and therefore we can fix a strictly increasing continuous sequence $A_{\xi} \quad\left(\xi<\omega_{1}\right)$ of countable subalgebras of $A$ whose union is equal to $A$. Let $X_{\xi}$ be the set of equivalence classes of the relation $E_{\xi}$ on $X$ defined by: $x E_{\xi} y$ iff there is no $a$ in $A_{\xi}$ containing $x$ but not $y$. Let $T$ be the union of $X_{\xi}$ for $\xi<\omega_{1}$. Then $T$ is a tree under the converse of inclusion. Note that, since every ideal is countably generated, $T$ cannot have uncountable branches. However, $T$ does have height exactly equal to $\omega_{1}$. For $t$ in $T$ let the height of $t$ be the maximal $\xi$ such that $t$ is an element of $X_{\xi}$. To every subset $U \subseteq T$ of elements of different heights we associate a naturally defined well-ordering: $t<u$ iff the height of $t$ is smaller than the height of $u$. The next lemma says that elements of $T$ behave much like the points of $X$.

Lemma 2 (PFA). Let $U$ be an uncountable set of elements of $T$ of different heights and for every $t$ in $U$ let $a_{t}$ be an element of $A$ containing $t$. Then there exist $t<u$ in $U$ such that $t \subseteq a_{u}$.

Proof. Suppose $t \nsubseteq a_{u}$ for all $t<u$ in $U$. We shall produce uncountable right-separated subsets of $U$ contradicting our assumption about $A$. We shall need the following combinatorial property of the sequence $a_{t}(t \in U)$.

Claim 1. Suppose $V \subseteq U$ is uncountable and that for some finite $n \geq 1$, $\mathscr{F} \subset[U]^{n}$ has order type $\omega_{1}^{n}$ under the lexicographical ordering induced by 
$<$. Then there exist $t$ in $V$ and $F$ in $\mathscr{F}$ above $t$ such that $t$ is not covered by $a_{u}(u \in F)$.

Proof. The proof is by induction on $n$. The case $n=1$ is our assumption about the sequence $a_{t}(t \in U)$. By throwing out a countable subset of $V$, we may assume that if an element of $A$ intersects an element of $V$ then it also intersects uncountably many of them. (We have used here the fact that the ideals of $A$ are countably generated.) By the induction hypothesis choose $v$ in $V$ and $F$ in $[U]^{n-1}$ such that:

(a) $\bar{v}=v \backslash \bigcup_{t \in F} a_{t}$ is not empty,

(b) $F \cup\{u\} \in \mathscr{F}$ for uncountably many $u$ in $U$

It suffices to find a $u$ as in (b) such that $\bar{v} \nsubseteq a_{u}$. If such a $u$ does not exist, since the complement of $\bar{v}$ is a countably generated ideal, there exist $a$ in $A$ containing $\bar{v}$ and uncountable $U_{0} \subseteq U$ such that $a \subseteq a_{u}$ for all $u$ in $U_{0}$. Choose $\xi$ such that $a \in A_{\xi}$. Since $a$ intersects an element of $V$, the set $V_{0}$ of all $v$ in $V$ of height $>\xi$ intersecting $a$ is uncountable. By the definition of $E_{\xi}$ it follows that $v \subseteq a$ for all $v$ in $V_{0}$. Pick $v_{0}$ in $V_{0}$ and $u$ in $U_{0}$ such that $v_{0}<u$. Then $v_{0} \subseteq a_{u}$, contradicting our initial assumption about $a_{t}$ $(t \in U)$. This proves the claim.

The fact that $A$ contains no uncountable right-separated subset means in particular that $X$ has a countable dense subset. Thus we may assume $A$ is a subalgebra of the power-set of the integers, so if $\theta$ is the successor of $2^{\aleph_{1}}$ all relevant objects are elements of the structure $H_{\theta}$. Our first poset $\mathscr{P}_{0}$ is the set of all pairs $p=\left\langle V_{p}, \mathscr{N}_{p}\right\rangle$ such that:

(c) $\mathscr{N}_{p}$ is a finite $\in$-chain of countable elementary submodels of the $H_{\theta}$ containing everything relevant,

(d) $V_{p}$ is a finite subset of $U$ separated by $\mathscr{N}_{p}$ such that no $t$ in $V_{p}$ is covered by the $a_{u}$ for $u>t$ in $V_{p}$.

( $V_{p}$ is separated by $\mathscr{N}_{p}$ means that for every $t<u$ in $V_{p}$ there is a model of $\mathscr{N}_{p}$ containing $t$ but not $u$.) The ordering of $\mathscr{P}_{0}$ is defined by letting $q \leq p$ if $V_{q}$ contains $V_{p}$ and $\mathscr{N}_{q}$ contains $\mathscr{N}_{p}$.

Claim 2. $\mathscr{P}_{0}$ is a proper poset.

Proof. Let $\kappa$ be a large enough regular cardinal and let $M$ be a countable elementary submodel of $H_{\kappa}$ containing $p$ and $\mathscr{P}_{0}$. Let

$$
q=\left\langle V_{p}, \mathscr{N}_{p} \cup\left\{M \cap H_{\theta}\right\}\right\rangle .
$$

Clearly, $q$ is an element of $\mathscr{P}_{0}$ and $q \leq p$. We shall prove that $q$ is an $M, \mathscr{P}_{0}$-generic condition. To this end, let $\mathscr{D} \in M$ be a dense open subset of $\mathscr{P}_{0}$ and let $r \leq q$. Extending $r$ we may assume that, in fact, $r$ is in $\mathscr{D}$. We need to find $\bar{r}$ in $\mathscr{D} \cap M$ compatible with $r$. Let $\bar{p}=r \cap M$. Note that $\bar{p}$ is in $\mathscr{P}_{0} \cap M$ and that it extends $p$. Let $F_{r}=V_{r} \backslash V_{\tilde{p}}$. We may assume this set is nonempty or else we are easily done. Let $n$ be the size of $F_{r}$. Note that every $t$ of $V_{\bar{p}}$ has a countable dense subset and, therefore, $t \cap M$ is dense in $t$. This means that for every $t$ in $V_{\bar{p}}$ we can fix $x_{t}$ in $t \cap M$ that is not in any $a_{u}$ for $u$ in $V_{r}$ above $t$. Let $\mathscr{F}$ be the set of all $F$ in $[U]^{n}$ such that for some $s$ in $\mathscr{D}$ end-extending $\bar{p}$ (i.e., $V_{\bar{p}}$ is an initial part of $V_{s}$ and $\mathscr{N}_{\bar{p}}$ is an initial part of $\left.\mathscr{N}_{s}\right), F$ is equal to $V_{s} \backslash V_{\bar{p}}$ and such that $x_{t}$ is not in $a_{u}$ for all $t$ in $V_{\bar{p}}$ and $u$ in $F$. Note that $\mathscr{F}$ is an element of $M \cap H_{\theta}$ and therefore an element of 
every model of $\mathscr{N}_{r}$ above $M \cap H_{\theta}$. Note also that $F_{r}$ is in $\mathscr{F}$. Using the fact that $F_{r}$ is separated by the models of $\mathscr{N}_{r}$ containing $\mathscr{F}$ one easily shows (see $[9, \S 8])$ that $\mathscr{F}$ must have order type $\omega_{1}^{n}$ under the lexicographical ordering induced by the well-ordering of $U$. For a family $\mathscr{G}$ of finite subsets of $U$ and $t$ in $U$, set

$$
\mathscr{G}_{t}=\left\{F \in[U]^{<\omega}:\{t\} \cup F \in \mathscr{G}\right\} .
$$

For a finite $F \subseteq U$, set $a(F)=\bigcup_{t \in F} a_{t}$. Finally, for $t$ in $U$, set

$$
W_{1}=\left\{t \in U: \mathscr{F}_{t} \text { has order type } \omega_{1}^{n-1}\right\} .
$$

Then $W_{1}$ is uncountable and it is an element of $M$. By removing countably many elements of $W_{1}$ we may assume that if an element of $A$ intersects an element of $W_{1}$ then it intersects uncountably many of them. Note that $t \nsubseteq a(F)$ for every $t$ in $W_{1}$ and $F$ in $\mathscr{F}_{t}$. Since $\omega_{1}^{n-1}$ cannot be covered by countably many sets of smaller order types, since every $t$ of $W_{1}$, as a subspace of $X$, has a countable dense subset, and since every point of $X$ is countably generated, to every $t$ of $W_{1}$ we can associate $b_{t}$ of $A$ intersecting $t$ and $\mathscr{G}_{t} \subseteq \mathscr{F}_{t}$ of order type $\omega_{1}^{n-1}$ such that $b_{t}$ is disjoint from $a(F)$ for every $F$ in $\mathscr{G}_{t}$. Clearly, we may assume that the mappings $t \mapsto b_{t}$ and $t \mapsto \mathscr{G}_{t}$ are both elements of $M$. Fix now a $t_{1}$ in $W_{1} \cap M$. We claim that $b_{t_{1}} \cap t_{1}$ cannot be a subset of $a\left(F_{r}\right)$. Otherwise, since the complement of $b_{t_{1}} \cap t_{1}$ is countably generated (and since it is an element of $M$ ) there is a $c$ in $A \cap M$ such that $b_{t_{1}} \cap t_{1} \subseteq c \subseteq a\left(F_{r}\right)$. Choose $\xi$ in $M$ such that $c \in A_{\xi}$. By our assumption the set $\bar{W}_{1}$ of all $w$ in $W_{1}$, such that $c \cap w \neq \varnothing$ and height $(w)>\xi$, is uncountable. By the definition of $E_{\xi}$, it follows that $w \subseteq c$ for all $w$ in $W_{1}$. Let

$$
\mathscr{H}=\left\{F \in[U]^{n}: c \subseteq a(F)\right\} .
$$

Then $\mathscr{H}$ is in $M \cap H_{\theta}$ and therefore is in any model of $\mathscr{N}_{r}$ above $M \cap H_{\theta}$. Since $F_{r}$ is in $\mathscr{H}$ and since it is separated by models of $\mathscr{N}_{r}$ above $M \cap H_{\theta}$ it follows easily that $\mathscr{H}$ has order type $\omega_{1}^{n}$ under the lexicographical ordering. Note that for every $w$ in $\bar{W}_{1}$ and $F$ in $\mathscr{H}$, we have $w \subseteq c \subseteq a(F)$, contradicting Claim 1. This proves that $b_{t_{1}} \cap t_{1}$ is not a subset of $a\left(F_{r}\right)$ and that therefore, for every $F$ in $\mathscr{G}_{t_{1}},\left\{t_{1}\right\} \cup F \cup F_{r}$ satisfies condition (d) for $t=t_{1}$. Now, working in $M$ with $\mathscr{G}_{t_{1}}$ instead of $\mathscr{F}$, we find $t_{2}$ in $M \cap U$ and $\mathscr{G}_{t_{1} t_{2}} \subseteq\left(\mathscr{G}_{t_{1}}\right)_{t_{2}}$ in $M$ of order type $\omega_{1}^{n-2}$ such that for all $F$ in $\mathscr{G}_{t_{1} t_{2}},\left\{t_{1}, t_{2}\right\} \cup F \cup F_{r}$ satisfies (d) for $t=t_{1}$ and $t=t_{2}$, etc. This procedure will give us $\left\{t_{1}, \ldots, t_{n}\right\}$ in $\mathscr{F} \cap M$ which together with $F_{r}$ satisfies condition (d). By the definition of $\mathscr{F}$, choose $\bar{r}$ in $\mathscr{D} \cap M$ end-extending $\bar{p}$ such that

$$
V_{\bar{r}} \backslash V_{\bar{p}}=\left\{t_{1}, \ldots, t_{n}\right\} .
$$

Then $r$ and $\bar{r}$ are compatible in $\mathscr{P}_{0}$. This finishes the proof of Claim 2.

Choose a countable elementary submodel $M$ of some large enough $H_{\kappa}$ containing $\mathscr{P}_{0}, H_{\theta}$, and all other relevant objects. Let $\bar{p}=\left\langle\{t\},\left\{M \cap H_{\theta}\right\rangle\right.$, where $t$ is an arbitrary element of $U \backslash M$. Then for all $\xi<\omega_{1}$,

$$
\mathscr{D}_{\xi}=\left\{p \in \mathscr{P}_{0}: \mathrm{ht}(t)>\xi \text { for some } t \in V_{p}\right\}
$$

is dense below $\bar{p}$. For suppose that for some $r \leq \bar{p}$ and $\xi<\omega_{1}$ there is no $q \leq r$ containing an element of $U$ of height $>\xi$. Let $\mathscr{E}$ be the set of all extensions $\bar{r}$ of $r \cap M$ for which there is an $\eta<\omega_{1}$ such that no $q \leq \bar{r}$ has a 
node of height $>\eta$. Then $\mathscr{E}$ is in $M$ and $r$ is an element of $\mathscr{E}$. The proof of Claim 2 shows that $\bar{p}$ is an $M, \mathscr{P}_{0}$-generic condition, so there must be an $\bar{r}$ in $\mathscr{E} \cap M$ compatible with $r$. So, choose $q \leq \bar{r}, r$. By the elementarity of $M$ there is an ordinal $\eta$ in $\omega_{1} \cap M$ witnessing the fact that $\bar{r}$ is in $\mathscr{E}$. But this is a contradiction, since $q$ is an extension of $\bar{r}$ which has the node $t$ of height $>\eta$. So we can choose a filter of $\mathscr{P}_{0}$ intersecting all $\mathscr{D}_{\xi}$ which will clearly give us an uncountable right-separated subset $V$ of $U$. This completes the proof of Lemma 2.

We are now ready for the final step of the proof of Theorem 1. For this we choose a sequence $c_{t} \quad(t \in U)$ of elements of $A$ such that:

(e) $U$ is an uncountable set of elements of $T$ of different heights,

(f) $c_{t} \cap t$ and $t \backslash c_{t}$ are both nonempty for all $t$ in $U$,

(g) for $t<u$ (i.e., $\mathrm{ht}(t)<\mathrm{ht}(u))$ in $U$ there is $\xi<\mathrm{ht}(u)$ such that $c_{t} \in A_{\xi}$.

Note that this means that for all $t<u$ in $U, c_{t}$ either contains or is disjoint from $u$. Our goal is to force uncountable $V \subseteq U$ such that for all $t<u$ in $V, c_{u}$ either contains or is disjoint from $t$. Hence for all $t$ in $V$ no element of $V \backslash\{t\}$ can split $t$; so, in particular, $c_{t}$ is not in the subalgebra generated by $c_{v} \quad(v \in V \backslash\{t\})$.

The poset $\mathscr{P}_{1}$ is the set of all pairs $p=\left\langle V_{p}, \mathscr{N}_{p}\right\rangle$ such that:

(h) $\mathscr{N}_{p}$ is a finite $\in$-chain of countable elementary submodels of $H_{\theta}$ containing everything relevant,

(i) $V_{p}$ is a finite subset of $U$ separated by $\mathscr{N}_{p}$ such that if $t<u$ are in $V_{p}$ then $c_{u}$ either contains or is disjoint from $t$.

For $p$ and $q$ in $\mathscr{P}_{1}$ set $q \leq p$ iff $V_{q} \supseteq V_{p}$ and $\mathscr{N}_{q} \supseteq \mathscr{N}_{p}$.

Claim 3. $\mathscr{P}_{1}$ is proper.

Proof. Let $\kappa$ be a large enough regular cardinal and let $M$ be a countable elementary submodel of $H_{\kappa}$ containing $p$ and $\mathscr{P}_{1}$. Let

$$
q=\left\langle V_{p}, \mathscr{N}_{p} \cup\left\{M \cap H_{\theta}\right\}\right\rangle \text {. }
$$

Then $q \in \mathscr{P}_{1}$ and $q \leq p$. We will show $q$ is $M, \mathscr{P}_{1}$-generic. So let $\mathscr{D} \in M$ be a dense open subset of $\mathscr{P}_{1}$ and let $r \leq q$ be given. We may assume $r$ is in $\mathscr{D}$ and we need to find $\bar{r}$ in $\mathscr{D} \cap M$ compatible with $r$. Let $\bar{p}=r \cap M$. Then $\bar{p} \in \mathscr{P}_{1} \cap M$. Let $F(r)=V_{r} \backslash V_{\bar{p}}$ and let $n$ be the size of $F(r)$. We may assume $n \geq 1$ since otherwise we are easily done. Let $\mathscr{F}$ be the set of all $F$ in $[U]^{n}$ for which there is an $s$ in $\mathscr{D}$ end-extending $\bar{p}$ such that $F=V_{s} \backslash V_{\bar{p}}$. Then $\mathscr{F}$ is an element of $M \cap H_{\theta}$ and therefore of every model of $\mathscr{N}_{r}$ above $M \cap H_{\theta}$. Since $F(r)$ is in $\mathscr{F}$, it follows as before that the order type of $\mathscr{F}$, $<_{\text {lex }}$, is equal to $\omega_{1}^{n}$. As before for $t$ in $U$, set

$$
\mathscr{F}_{t}=\left\{F \in[U]^{n-1}: F \cup\{t\} \in \mathscr{F}\right\} \text {. }
$$

Let

$$
W_{1}=\left\{t \in U: \mathscr{F}_{t} \text { has order type } \omega_{1}^{n-1}\right\}
$$

Then $W_{1}$ is uncountable and $W_{1}$ is in $M$. Choose $\xi$ such that $c_{u} \in A_{\xi}$ for all $u$ in $V_{r}$ and choose $w$ in $W_{1}$ of height $>\xi$. Then by the definition of the equivalence relation $E_{\xi}, w \subseteq c_{u}$ or $w \cap c_{u}=\varnothing$ for every $u$ in $V_{r}$. Choose 
$a_{w}$ in $A$ containing $w$ such that for every $u$ in $V_{r}, a_{w}$ is either included or is disjoint from $c_{u}$. Since $W_{1}$ is an element of the elementary submodel $M$, by Lemma 2 there is a $t_{1}$ in $W_{1} \cap M$ such that $t_{1} \subseteq a_{w}$. Note that this means that no $c_{u}$ for $u$ in $V_{r}$ splits $t_{1}$. Working with $\mathscr{F}_{t_{1}}$ instead of $\mathscr{F}$ we find $t_{2}$ in $U \cap M$ such that $\left(\mathscr{F}_{t_{1}}\right)_{t_{2}}$ has order type $\omega_{1}^{n-2}$ and such that no $c_{u}$ for $u$ in $V_{r}$ splits $t_{2}$, etc. This procedure will give us $\left\{t_{1}, \ldots, t_{n}\right\}$ in $\mathscr{F} \cap M$ such that none of the $t_{i}$ is split by any $c_{u}$ for $u$ in $V_{r}$. This means that $\left\{t_{1}, \ldots, t_{n}\right\} \cup V_{r}$ satisfies condition (i) from the definition of $\mathscr{P}_{1}$. By the definition of $\mathscr{F}$, there is an $\bar{r}$ in $\mathscr{D} \cap M$ such that $\left\{t_{1}, \ldots, t_{n}\right\}$ is equal to $V_{\bar{r}} \backslash V_{\bar{p}}$. It follows that $r$ and $\bar{r}$ are compatible in $\mathscr{P}_{1}$. This finishes the proof of Claim 3 .

Choose a countable elementary submodel $M$ of some large enough $H_{\kappa}$ containing everything relevant and set $\bar{p}=\left\langle\{t\},\left\{M \cap H_{\theta}\right\}\right\rangle$, where $t$ is any element of $U \backslash M$. Then for all $\xi<\omega_{1}$,

$$
\mathscr{D}_{\xi}=\left\{p \in \mathscr{P}_{1}: V_{p} \text { has an element of height }>\xi\right\}
$$

is dense below $\bar{p}$. Then a filter of $\mathscr{P}_{1}$ intersecting all these dense sets gives the uncountable irredundant subset of $A$ we have been searching for. This finishes the proof of Theorem 1.

Remark 1. The final step of the proof of Theorem 1 can also be done via the partition property $(P)$ already used above in Lemma 1 . Again we start with a sequence $c_{t}(t \in U)$ of elements of $A$ with properties (e), (f), and (g). Define $[U]^{2}=K_{0} \cup K_{1}$ by letting $\{t, u\}_{<}$in $K_{0}$ iff $c_{u}$ either contains or is disjoint from $t$. The partition property $(\mathbf{P})$ of $[9, \S 9]$ says that either there is uncountable $V \subseteq U$ such that $[V]^{2} \subseteq K_{0}$, or else there is uncountable $W \subseteq U$ and disjoint uncountable $\mathscr{F} \subseteq[U]^{<\omega}$ such that if $t$ is in $W$ and $F$ is in $\mathscr{F}$ above $t$ then there is $u$ in $F$ such that $\{t, u\}$ is an element of $K_{1}$, i.e., $c_{u}$ splits $t$. Note that the first alternative is just what we want, i.e., $c_{v}$ for $v$ in $V$ forms an uncountable irredundant subset of $A$. So it suffices to show that the second alternative cannot happen. This will be done using Lemma 2. For each $F$ in $\mathscr{F}$ let $t_{F}$ be the minimal element of $W$ that is above every element of $F$. By the definition of equivalence relations $E_{\xi}$ (and by $\left.(\mathrm{g})\right)$ this means that $t_{F}$ is either contained or is disjoint from $c_{u}$ for every $u$ in $F$. Let

$$
a_{F}=\bigcap_{u \in F} \varepsilon_{u} c_{u},
$$

where $\varepsilon_{u} c_{u}=c_{u}$ if $c_{u}$ contains $t_{F}$ and $\varepsilon_{u} c_{u}=-c_{u}$ if $c_{u}$ is disjoint from $t_{F}$. Note that $t_{F} \subseteq a_{F}$. Refining $\mathscr{F}$, we may assume that $t_{F}<G$ for $F<G$ in $F$. By Lemma 2 there exist $F<G$ in $F$ such that $t_{F} \subseteq a_{G}$. This means that no $c_{u}$ for $u$ in $G$ splits $t_{F}$, contradicting the property of $W$ and $\mathscr{F}$.

Remark 2. The proof of Theorem 1 actually gives the following apparently stronger conclusion: Every Boolean algebra $A$ of size $\aleph_{1}$ either has an uncountable ideal-independent set or is the union of countably many irredundant sets. To see this note that $A$ is the union of countably many sets having at most one point in each of the differences $A_{\xi+1} \backslash A_{\xi}$ for $\xi<\omega_{1}$, so it suffices to decompose any such $J \subseteq A$ into countably many irredundant sets. For $c$ in $J$, let $\xi$ be such that $c$ is an element of $A_{\xi+1} \backslash A_{\xi}$. Then there must be $t$ in $X_{\xi}$ such that $c \cap t$ and $t \backslash c$ are both nonempty. Hence, we may assume that 
$J$ is equal to some $c_{t}(t \in U)$ with properties (e), (f), and (g). Now consider the partition $[U]^{2}=K_{0} \cup K_{1}$ of Remark 1, i.e., we put $\{t, u\}_{<}$in $K_{0}$ iff $c_{u}$ does not split $t$. The proof from Remark 1 actually shows (with the help of the partition property $(\mathrm{P})$ ) that the poset $\mathscr{P}$ of all finite 0 -homogeneous sets is ccc. An applicaton of $\mathrm{MA}_{\aleph_{1}}$ to $\mathscr{P}<\omega$ gives us a decomposition of $U$ into countably many 0 -homogeneous sets, so we are done.

Remark 3. The conclusion of Theorem 1 can also be extended (without additional work) to a larger class of algebras that includes all varieties generated by single finite primal algebras, i.e., algebras having all functions representable by terms. This is so since every algebra $B$ from such a variety is isomorphic to a Boolean power of the generating algebra, so the irredundant set of the corresponding Boolean algebra transfers to an irredundant set of $B$. This answers a question asked in [4] where we refer the reader for more information about the existence of irredundant sets in the setting of universal algebra.

Our second result about the problem of irredundance shows that the statement from Theorem 1 has a considerable influence on the real numbers and that in some sense the axiomatic approach to the problem was necessary. It should be noted that at the time the problem of irredundance was asked in [2] it was known that it cannot be solved using the usual axioms of set theory. That observation depended on an example of M. Rubin from [6]. Our proof will use the following observation of L. Heindorf [4] which gives a proper formulation to an old argument (see [10, p. 237] for an explanation of this point).

Lemma 3. If $I$ is an irredundant subset of a Boolean algebra $A$ then $\{a \times-a$ : $a \in I\}$ is ideal-independent in the free product $A * A$.

Proof. Let $F$ be a finite subset of $I$ and let $a$ be an element of $I$ outside $F$. Since $a$ is not in the subalgebra $B$ generated by $F$, there is an atom $c$ of $B$ such that $a \cap c$ and $c \backslash a$ are both nonempty. Then $(a \cap c) \times(c \backslash a)$ is a nonempty subset of $a \times-a$ that is disjoint from every $b \times-b$ for $b$ in $F$. Thus, $a \times-a$ is not a subset of the union of $b \times-b$ for $b$ in $F$. This finishes the proof.

Note that the dual of an ideal-independent set is a discrete subspace of the dual space. Note also that the dual space of $A * A$ is the square of the dual space of $A$. This enables us to state the result of $[9, \S 2]$ in the following form relevant to the present context.

Theorem 2. If every uncountable Boolean algebra contains an uncountable irredundant set then every subset of $\omega^{\omega}$ of size $\aleph_{1}$ is bounded in the ordering of eventual dominance.

Proof. Suppose there is an $X \subseteq \omega^{\omega}$ of size $\aleph_{1}$ unbounded under $<^{*}$. In [9, 2.4] we have refined its topology to a locally countable locally compact topology with no uncountable discrete subset in any finite power. Let $A$ be the subalgebra of the clopen algebra of $X$ generated by compact open sets. Since the dual space of $A$ is the one point compactification of $X$, by Lemma 3 it follows that $A$ has no uncountable irredundant subset. This finishes the proof.

The problem of irredundance versus the size will now be considered using the terminology of cardinal invariants [5]. So, let $\operatorname{irr}(A)$ be the supremum of cardinalities of irredundant subsets of $A$ and let $\operatorname{ig}(A)$ be the minimal $\theta$ such 
that every ideal of $A$ is generated by $\leq \theta$ elements. The function ig is the dual of a well-known cardinal function on topological spaces while irr seems to be purely algebraic. The next result shows that $\operatorname{irr}(A)$ and $\operatorname{ig}(A)$ restrict the size of $A$ in an interesting way.

Proposition 1. $\operatorname{Card}(A) \leq \operatorname{ig}(A)^{+} \cdot \operatorname{irr}(A)$.

Proof. Let $\theta=\operatorname{ig}(A)^{+} \cdot \operatorname{irr}(A)$ and suppose that $A$ has cardinality $>\theta$. We may assume that, in fact, $A$ has size $\theta^{+}$. Decompose $A$ into a strictly increasing continuous sequence $A_{\xi}\left(\xi<\theta^{+}\right)$of subalgebras of size $\leq \theta$. Let $\kappa=\operatorname{ig}(A)^{+}$ and for every $\delta<\theta^{+}$of cofinality $\kappa$ choose $a_{\delta}$ in $A_{\delta+1} \backslash A_{\delta}$. Let

$$
I_{\delta}=\left\{b \in A_{\delta}: b \cap a_{\delta} \in A_{\delta}\right\}
$$

be the ideal of $A_{\delta}$ determined by $a_{\delta}$. By the choice of $\kappa$, for every such $\delta$ there is $\xi=f(\delta)$ such that $I_{\delta} \cap A_{\xi}$ generates $I_{\delta}$. By the Pressing Down Lemma there exist $\xi_{0}$ and stationary $S \subseteq \theta^{+}$of cofinality $\kappa$ ordinals such that $f(\delta)=\xi_{0}$ for all $\delta$ in $S$. We claim that $\left\{a_{\delta}: \delta \in S\right\}$ is irredundant in $A$, which gives a contradiction that finishes the proof. This will follow easily from the following fact, where $\varepsilon a=a$ for $\varepsilon=+$ and $\varepsilon a=-a$ for $\varepsilon=-$.

Claim 4. Suppose $\delta<\delta_{0}<\cdots<\delta_{n}$ are elements of $S$ and the $\varepsilon_{i}(i \leq n)$ are elements of $\{+,-\}$. If for some $c$ in $A_{\delta}$

$$
c \cap \bigcap_{i \leq n} \varepsilon_{i} a_{\delta_{i}} \subseteq a_{\delta},
$$

then there exist $b_{0}, \ldots, b_{n}$ in $A_{\xi_{0}}$ such that

$$
c \cap \bigcap_{i \leq n} \varepsilon_{i} a_{\delta_{i}} \subseteq c \cap \bigcap_{i \leq n} b_{i} \subseteq a_{\delta} .
$$

Proof. The proof is by induction on $n$. Let

$$
d=\left(c \cap \bigcap_{i<n} \varepsilon_{i} a_{\delta_{i}}\right) \backslash a_{\delta}
$$

Then $d \in A_{\delta_{n}}$ and $d \subset-\varepsilon_{n} a_{\delta_{n}}$. Since $I_{\delta_{n}} \cap A_{\xi_{0}}$ generates $I_{\delta_{n}}$, there is a $b_{n}$ in $A_{\xi_{0}}$ such that $d \subseteq-b_{n} \subseteq-\varepsilon_{n} a_{\delta_{n}}$. Then

$$
c \cap \bigcap_{i \leq n} \varepsilon_{i} a_{\delta_{i}} \subseteq c \cap b_{n} \cap \bigcap_{i<n} \varepsilon_{i} a_{\delta_{i}} \subseteq a_{\delta},
$$

so we are done by the induction hypothesis.

If $\left\{a_{\delta}: \delta \in S\right\}$ is not irredundant we can find $\delta<\delta_{0}<\cdots<\delta_{n}$ such that $a_{\delta}$ is generated by $A_{\delta} \cup\left\{a_{\delta_{0}}, \ldots, a_{\delta_{n}}\right\}$. Considering the disjunctive normal form it follows that $a_{\delta}$ is the union of elements of the form $c \cap \bigcap_{i \leq n} \varepsilon_{i} a_{\delta_{i}}$, where $c \in A_{\delta}$. By Claim 4 every such intersection can be replaced by one of the form

$$
c \cap \bigcap_{i \leq n} b_{n}
$$

for some $b_{0}, \ldots, b_{n}$ in $A_{\xi_{0}}$. It follows that $a_{\delta}$ is in the subalgebra $A_{\delta}$, a contradiction. This finishes the proof of Proposition 1.

An example of Rubin [6] shows that the successor in Proposition 1 is necessary. However, the proposition suggests the following questions. 
Question 1. Is ig or Card bounded by irr $^{+}$?

Question 2. Is the weight of a compact space $X$ bounded by $s\left(X^{2}\right)^{+}$?

Note that Proposition 1 shows that under MA $_{\aleph_{1}}$ every BA of countable irredundance has size at most $\aleph_{1}$. Another application of Proposition 1 shows that if we add a number of Cohen reals then every algebra of countable irredundance has size bounded by the continuum of the ground model. This uses an argument involving the homogeneity of the Cohen poset which will be presented in the proof of the following proposition.

Proposition 2. If $\theta$ is inaccessible and if we add $\theta$ Cohen reals then in the extension every $B A$ of size continuum has an irredundant subset of size continuum.

Proof. Since the subalgebra of $\dot{A}$ generated by a maximal irredundant subset of $\dot{A}$ is dense in $\dot{A}$, we may assume that $\dot{A}$ has a dense subset of size $\kappa<\theta$. Hence we may assume $\dot{A}$ is a subalgebra of the power-set algebra of $\kappa$. Choose names $\dot{a}_{\xi}(\xi<\theta)$ for subsets of $\kappa$ which are forced to be elements of $\dot{A}$ with the property that $\dot{a}_{\eta}$ is not in the subalgebra generated by $\dot{a}_{\xi}$ for $\xi<\eta$. Using the inaccessibility of $\theta$ and refining the sequence of names, we may assume that the domains of the $\dot{a}_{\xi}$ 's form a $\Delta$-system and that they are all isomorphic modulo the order-isomorphism of their domains. We claim now that no condition $p$ can force some $\dot{a}_{\eta}$ to be generated by $\dot{a}_{\xi}(\xi \in F)$, where $F$ is a finite subset of $\theta \backslash\{\eta\}$. This is so since we can find $\bar{F}$ and $\bar{\eta}$ with $\bar{\eta}$ above $\bar{F}$ such that an automorphism of the Cohen poset transforms $\dot{a}_{\eta}$ to $\dot{a}_{\bar{\eta}}$ and $\dot{a}_{\xi}(\xi \in F)$ to $\dot{a}_{\bar{\xi}}(\bar{\xi} \in \bar{F})$. This would mean that some condition $\bar{p}$ forces $\dot{a}_{\bar{\eta}}$ to be generated by $a_{\bar{\xi}}(\bar{\xi} \in \bar{F})$, contradicting our initial assumption. This completes the proof.

Concerning Proposition 2 we mention a result of Devlin [1] that if there is a $\sigma$-saturated $\kappa$-complete ideal on $\kappa$ then every (Boolean) algebra of size $\kappa$ has an irredundant subset of size $\kappa$. This gives us some restrictions to any use of ccc forcing in order to provide a negative answer to Question 1.

The axiom used in Theorem 1 has some large cardinal strength so the readers interested in consistency results may ask whether the consistency of the statement can be proved without any use of large cardinals. This can indeed be proved using the method described in $[7 ; 8 ; 9, \S 8]$, where the $\in$-chains of submodels are replaced by matrices of isomorphic submodels giving us the strong chain condition to the posets forcing uncountable irredundant sets. The details of this are left to the interested reader who may wish to consult [8], where a similar proof is presented.

\section{REFERENCES}

1. K. J. Devlin, Some weak versions of large cardinal axioms, Ann. of Math. Logic 4 (1973), 291-326.

2. E. K. van Douwen, J. D. Monk, and M. Rubin, Some questions about Boolean algebras, Algebra Universalis 11 (1980), 220-243.

3. G. Gruenhage, On the existence of metrizable or Sorgenfrey subspace, Proc. Sixth Prague Topol. Symposium 1986 (Z. Frolik, ed.), Heldermann-Verlag, Berlin, 1988, pp. 223-230.

4. L. Heindorf, A note on irredundant sets, Algebra Universalis 26 (1989), 216-221. 
5. J. D. Monk, Cardinal functions on Boolean algebras, Lectures in Math., ETH Zürich, Birkhäuser, 1990.

6. M. Rubin, A Boolean algebra with few subalgebras, interval algebras and retractiveness, Trans. Amer. Math. Soc. 278 (1983), 65-89.

7. S. Todorcevic, A note on the Proper Forcing Axiom, Contemp. Math. 31 (1984), 209-218.

8. __ Directed sets and cofinal types, Trans. Amer. Math. Soc. 290 (1985), 711-729.

9. __ Partition problems in topology, Amer. Math. Soc., Providence, R.I.,, 1988.

10. __ Free sequences, Topology Appl. 35 (1980), 235-238.

Mathematical Sciences Research Institute, 1000 Centennial Drive, Berkeley, CaliFORNIA 94720

Current address: Matematički Institut, Kneza Mihaila 35, 11001 Beograd, P.P. 367, Yugoslavia and Department of Mathematics, University of Toronto, Toronto, Ontario, Canada M5S 1A1

E-mail address: stevo@math.toronto.edu 